\title{
MECHANICAL CHAINS: IDENTIFICATION OF DYNAMICAL RESPONSES
}

\author{
A.I. Artyunin' ${ }^{I}$ S.K. Kargapoltsev ${ }^{I}$, S.V. Eliseev ${ }^{I}$ and R.S. Bolshakov ${ }^{I}$
}

1 Irkutsk State Transport University, Irkutsk, Russia Federation e-mail: artyunin_ai@irgups.ru

\begin{abstract}
Approaches for dynamical responses identification in example of system with three freedom degrees with tie between two of outside elements are offered. First approach bases on using of mathematical models-analogues of automation control theory, second approach bases on rules of transformation of design schemes of chains theory. Conversion from design schemes to equivalent in dynamical attitude of structural schemes of automation control is applied at using of structural method. Feedback tie in automation control system dedicated to the protection object is dynamical responses on external disturbance and at zero value of the disturbance disposed of the coerced elasticity of the system. The using of direct conversion of design schemes for the rules of chains theory shows identical results for both approaches.
\end{abstract}

Keyboards: dynamical responses, structural approaches, chains theory, feedback ties, coerced elasticity;

\section{INTRODUCTION}

Vibroprotection systems (VPS) of technical objects are various enough and can contain in the structure various elements which often unite in mechanical chains. At presence in chains of inertial elements and periodic external influences dynamic interactions form the responses of communications defining a condition of object of protection, contacts to basic surfaces and level of power interactions between elements $[3 \div 5]$.

Dynamic esponses of communication are simply enough defined in flat mechanisms which represent the closed mechanical chains with one or several motionless links. Kinetostatical calculations and methodical base of calculations are well enough developed and have found reflexion in works of domestic scientists $[1,7,9]$.

At the same time, definition of responses of communications in mechanical oscillatory systems is developed to a lesser degree. Though to questions of dynamics of oscillative motions in mechanisms [7,8] and vibroprotection systems the considerable attention $[9,10]$ is paid.

Specificity vibroprotection systems consists in prevalence of periodic forms of movements of object of protection and links ÂÇÑ that in practice often leads to disclosing of contacts, sample of backlashes and occurrence vibroimpact processes that is undesirable effects in respect of maintenance of reliability and 
safety of operation of cars, the equipment and apparatus $[11,12]$.

In article the methodological basis of definition of dynamic reactions in mechanical oscillatory systems with use of the structural mathematical models-analogues received by transformation by Laplace initial linear or linearized of the differential equations of movement is offered.

I. Formulation of the tasks. Consider as initial design scheme of vibroprotection system in view of mechanical oscillation system with three freedom degree (fig. 1). The system makes small movements under the influence of periodical and kinematical disturbances which can be applied to all mass-inertial elements $Q_{i}(i=\overline{1,3})$.
Besides accounting of action of kinematical disturbances $z_{k}(k=\overline{1,2})$, which connected with bearing surfaces $I$ and $I I$ (fig. 1), is possible. The fixed coordinate system $y_{1}, y_{2}, y_{3}$ is selected as the main; friction forces are considered negligible and elements movements with masses $\mathrm{m}_{1}, \mathrm{~m}_{2}, \mathrm{~m}_{3}$ occur on vertical; stiffness coefficients of linear elastical elements are designated through $k_{1}, k_{2}, k_{3}, k_{4}, k_{5}$. Circuit $I$ on fig. 1 determines placement of vibroprotection device (VPD); protection object has a mass $\mathrm{m} 3$. Bearing surface $I I$ is introduced for creature more full scheme: at necessity $k_{4}$ and $k_{5}$, as well as $Q_{i}$ and $z_{k}$, may take zero values. Write equations for kinetical and potential energies:

$$
\begin{aligned}
& T=\frac{1}{2} m_{1} \dot{y}_{1}^{2}+\frac{1}{2} m_{2} \dot{y}_{2}^{2}+\frac{1}{2} m_{3} \dot{y}_{3}^{2} \\
& \Pi=\frac{1}{2} k_{1} y_{1}^{2}+\frac{1}{2} k_{2}\left(y_{2}-y_{1}\right)^{2}+\frac{1}{2} k_{3}\left(y_{3}-y_{2}\right)^{2}+\frac{1}{2} k_{4} y_{3}^{2}+\frac{1}{2} k_{5}\left(y_{3}-y_{1}\right)^{2}
\end{aligned}
$$

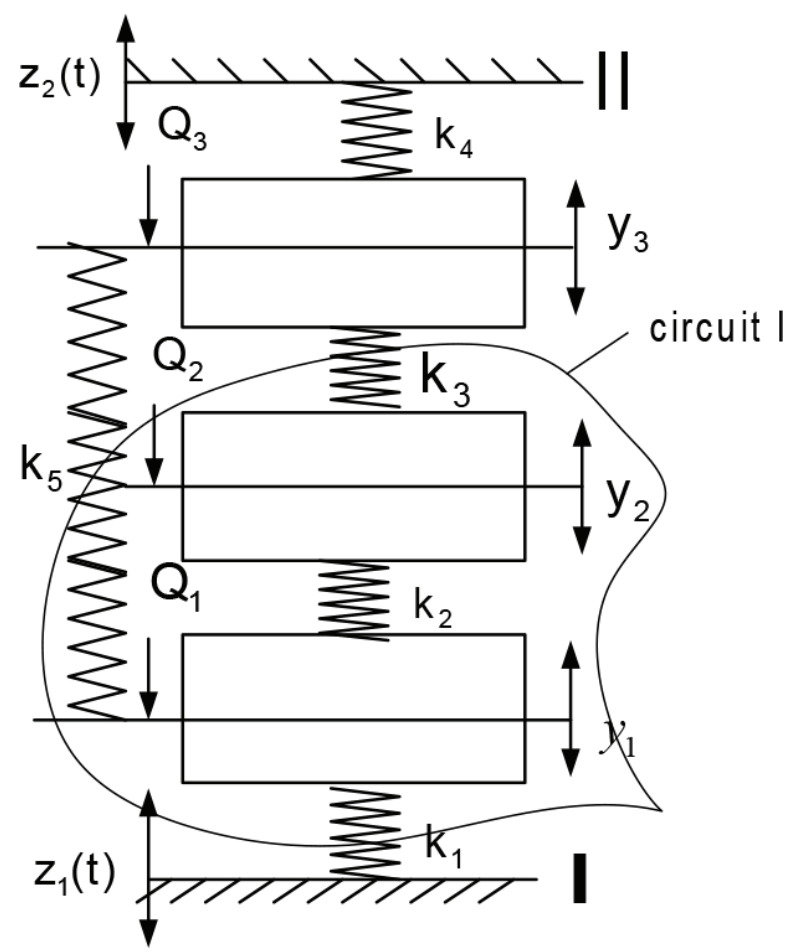

Fig. 1. Design scheme of vibroprotection systems with three freedom degrees 
For construction of mathematical model

the standard technique [5] on the basis of a

formalism of LaGrange is used:

$$
\begin{aligned}
& \mathrm{m}_{1} \ddot{\mathrm{y}}_{1}+\mathrm{y}_{1}\left(\mathrm{k}_{1}+\mathrm{k}_{2}+\mathrm{k}_{3}\right)-\mathrm{k}_{2} \mathrm{y}_{2}-\mathrm{k}_{5} \mathrm{y}_{3}=\mathrm{k}_{1} \mathrm{z}_{1} \\
& \mathrm{~m}_{2} \ddot{\mathrm{y}}_{2}+\mathrm{y}_{2}\left(\mathrm{k}_{2}+\mathrm{k}_{3}\right)-\mathrm{k}_{2} \mathrm{y}_{1}-\mathrm{k}_{5} \mathrm{y}_{3}=0 \\
& \mathrm{~m}_{3} \ddot{\mathrm{y}}_{3}+\mathrm{y}_{3}\left(\mathrm{k}_{3}+\mathrm{k}_{4}+\mathrm{k}_{5}\right)-\mathrm{k}_{3} \mathrm{y}_{2}-\mathrm{k}_{5} \mathrm{y}_{1}=\mathrm{Q}_{3}
\end{aligned}
$$

If to accept that $\mathrm{z}_{1}=0, \mathrm{z}_{2}=0$, and $\mathrm{Q}_{1} \neq 0, \mathrm{Q}_{2} \neq$ can be presented in the form of following $0, \mathrm{Q}_{3} \neq 0$, communications between variables equations: $\mathrm{y}_{1}, \mathrm{y}_{2}, \mathrm{y}_{3}$ and $\mathrm{Q}_{1}, \mathrm{Q}_{2}, \mathrm{Q}_{3}$ in the operational form

$$
\begin{aligned}
& \tilde{y}_{1}=\frac{\tilde{Q}_{1}\left(a_{22} a_{33}-a_{23} a_{32}\right)+\tilde{Q}_{2}\left(a_{13} a_{32}-a_{33} a_{12}\right)+\tilde{Q}_{3}\left(a_{12} a_{23}-a_{13} a_{22}\right)}{A_{0}}, \\
& \tilde{y}_{2}=\frac{\tilde{Q}_{1}\left(a_{23} a_{31}-a_{31} a_{33}\right)+\tilde{Q}_{2}\left(a_{11} a_{33}-a_{13} a_{31}\right)+\tilde{Q}_{3}\left(a_{13} a_{21}-a_{11} a_{23}\right)}{A_{0}}, \\
& \tilde{y}_{3}=\frac{\tilde{Q}_{1}\left(a_{21} a_{32}-a_{22} a_{31}\right)+\tilde{Q}_{2}\left(a_{12} a_{31}-a_{11} a_{32}\right)+\tilde{Q}_{3}\left(a_{11} a_{22}-a_{12} a_{21}\right)}{A_{0}},
\end{aligned}
$$

where

$$
\begin{aligned}
& A_{0}=a_{11} a_{22} a_{33}-a_{11} a_{23} a_{32}-a_{33} a_{12} a_{21}- \\
& -a_{22} a_{13} a_{31}+a_{13} a_{21} a_{32}+a_{12} a_{23} a_{31} .
\end{aligned}
$$

Is the characteristic equation of system (the system is symmetric: $\mathrm{a}_{12}=\mathrm{a}_{21}, \mathrm{a}_{13}=\mathrm{a}_{31}, \mathrm{a}_{23}=\mathrm{a}_{32}$ ); the badge $(\sim)$ means the image on Laplace as in relation to $(3) \div(5)$ transformation of Laplace is applied to the further calculations.

Using $(6) \div(8)$, it is possible to construct enough great number of transfer functions.
For the further consideration it is accepted that on object of protection external periodic (harmonious) force $\mathrm{Q}_{3} \neq 0$ operates, other power factors are equal 0 . The structural scheme of system corresponding to the equations $(3) \div(5)$ is resulted on fig. 2 .

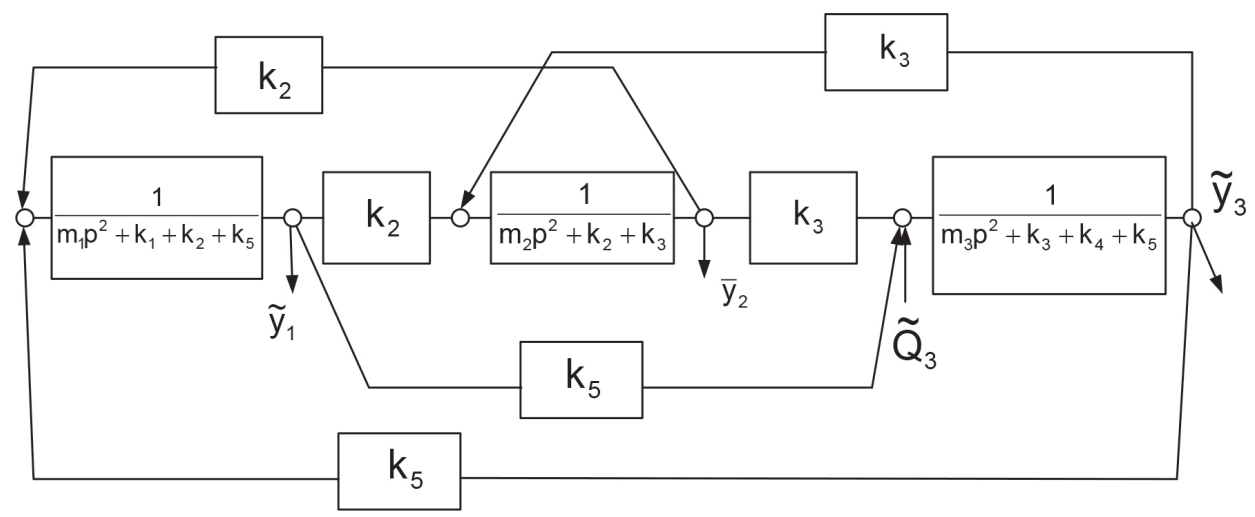

Fig. 2. Structural scheme for vibroprotection system on fig. 1 at $\tilde{\mathbf{Q}}_{3} \neq 0$ 
At action of external indignations dynamic responses arise between object of protection $\mathrm{m}_{3}$ and a support $I I$, and also between an inertial intermediate element $\mathrm{m}_{1} \mathrm{VPD}$ and a basic surface $I$. Dynamic responses will arise as well between separate elements of system.

\section{Estimation of dynamical properties} of system. Using equations $(6) \div(8)$, we will write down for structural system on fig. 2 transfer functions:

$$
\begin{aligned}
& W_{1}(p)=\frac{\tilde{y}_{1}}{\tilde{Q}_{3}}=\frac{a_{12} a_{23}-a_{13} a_{22}}{A_{0}} ; \\
& W_{2}(p)=\frac{\tilde{y}_{2}}{\tilde{Q}_{3}}=\frac{a_{13} a_{21}-a_{11} a_{23}}{A_{0}} ; \\
& W_{3}(p)=\frac{\tilde{y}_{3}}{\tilde{Q}_{3}}=\frac{a_{11} a_{22}-a_{12} a_{21}}{A_{0}}
\end{aligned}
$$

where $A_{0}$ it is defined by equation (9). As the structural scheme on fig. 2 has communication between co-ordinates $\mathrm{y}_{1}$ and $\mathrm{y}_{3}\left(\mathrm{k}_{5} \neq 0\right)$ a particular interest represents a case loading $\mathrm{Q}_{2} \neq 0$. We will write down transfer functions of system for this case:

$$
\begin{aligned}
& W_{1}^{\prime}(p)=\frac{\tilde{y}_{1}}{\tilde{Q}_{2}}=\frac{a_{13} a_{32}-a_{12} a_{33}}{A_{0}} ; \\
& W_{2}^{\prime}(p)=\frac{\tilde{y}_{2}}{\tilde{Q}_{2}}=\frac{a_{11} a_{33}-a_{13} a_{31}}{A_{0}} ; \\
& W_{3}^{\prime}(p)=\frac{\tilde{y}_{3}}{\widetilde{Q}_{2}}=\frac{a_{12} a_{31}-a_{11} a_{32}}{A_{0}} .
\end{aligned}
$$

Equations (10) $\div$ (15) for transfer functions can be used for structural scheme transformation on fig. 2.

1. Let's consider a case $\widetilde{Q}_{3} \neq 0$ then (12) it is possible to transform to a kind

$$
\begin{gathered}
W_{3}(p)=\frac{\widetilde{y}_{3}}{\widetilde{Q}_{3}}=\frac{1}{a_{33}-\frac{a_{11} a_{23}^{2}}{A_{1}}-. .} \\
\ldots \frac{-\frac{a_{22} a_{13}^{2}}{A_{1}}+\frac{2 a_{12} a_{23} a_{31}}{A_{1}}}{,}
\end{gathered}
$$

$$
\text { where } A_{1}=a_{11} a_{22}-a_{12}^{2} \text {. }
$$

The detailed view (16) takes the form:

$$
\begin{aligned}
& W_{3}^{*}(p)=\frac{\widetilde{y}_{3}}{\widetilde{Q}_{3}}=\frac{1}{m_{3} p^{2}+k_{3}+k_{4}+k_{5}-\ldots} \\
&-\frac{k_{5}^{2}\left(m_{2} p^{2}+k_{2}+k_{3}\right)}{A_{2}}-\ldots
\end{aligned}
$$

$$
\ldots-\frac{k_{3}^{2}\left(m_{1} p^{2}+k_{1}+k_{2}+k_{5}\right)}{A_{2}}--\frac{2 k_{5} k_{2} k_{3}}{A_{2}} .
$$

Here

$$
A_{2}=\left(m_{1} p^{2}+k_{1}+k_{2}+k_{5}\right)\left(m_{2} p^{2}+k_{2}+k_{3}\right)-k_{2}^{2},\left(18^{\prime}\right)
$$
and $p=j \omega-$ complex variable $(j=\sqrt{ }-1)$.

In turn, on equation (18) the structural scheme, as is shown in fig. 3 can be constructed. On this scheme the structural element corresponding to object of protection $\mathrm{m} 3$ is allocated. All other elements are grouped in a chain of feedback which, on the physical essence, represents the generalized spring with the stiffness defined by equation:

$$
\begin{aligned}
\tilde{k}_{c}^{\prime}= & \left(k_{3}+k_{4}+k_{5}\right) A_{2}-\frac{k_{5}^{2}\left(m_{2} p^{2}+k_{2}+k_{3}\right)+\ldots}{A_{2}} \\
& \ldots+k_{3}^{2}\left(m_{1} p^{2}+k_{1}+k_{2}+k_{5}\right)-2 k_{2} k_{3} k_{5} .
\end{aligned}
$$

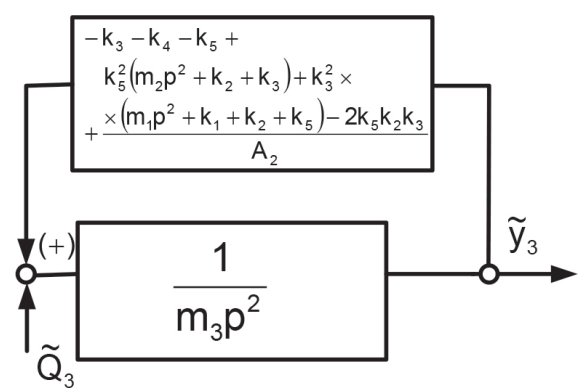

Fig. 3. Coerced structural scheme for identification dynamical response on protection object

Let's notice that feedback in the structural scheme (fig. 3) will have a positive sign. Dimension of an entrance signal in a return chain corresponds to displacement, and the target signal has dimension of force. That is feedback, in physical sense, reflects interaction with a basic surface II that corresponds to dynamic response 


$$
\tilde{R}_{B}=k_{4} \tilde{y}_{3}=k_{4} W_{3}(p) \tilde{Q}_{3} .
$$

The second part of dynamic response is defined by interaction of elastic elements $k_{4}$ and $\mathrm{k}_{5}$ and also an additional member who in equation (19) has a negative sign:

$$
\begin{aligned}
& \tilde{R}_{m_{3}}=\left[\frac{\left(k_{4}+k_{3}+k_{5}\right) A_{2}-k_{5}^{2}\left(m_{2} p^{2}+k_{2}+k_{3}\right)+\ldots}{A_{2}}\right] \\
& \ldots+k_{3}^{2}\left(m_{1} p^{2}+k_{1}+k_{2}+k_{5}\right)-2 k_{2} k_{3} k_{5} \\
& y_{3} .
\end{aligned}
$$

Transfer function at a power input $\mathrm{Q}_{3}$ and an exit $-\tilde{R}_{m_{2}}$ has view:

$$
\begin{aligned}
W_{m_{3}}(p) & =\frac{\widetilde{R}_{m_{3}}}{\widetilde{Q}_{3}}=\frac{\left(k_{3}+k_{4}+k_{5}\right) A_{2}-}{A_{0}} \ldots \\
\ldots & -\left[k_{5}^{2}\left(m_{2} p^{2}+k_{2}+k_{3}\right)+\right.
\end{aligned}
$$$$
\left.. .+\mathrm{k}_{3}^{2}\left(\mathrm{~m}_{1} \mathrm{p}^{2}+\mathrm{k}_{1}+\mathrm{k}_{2}+\mathrm{k}_{5}\right)-2 \mathrm{k}_{2} \mathrm{k}_{3} \mathrm{k}_{5}\right]_{\mathrm{W}_{3}(\mathrm{p})}
$$

where

$$
\begin{aligned}
& W_{3}(p)=\frac{1}{A_{0}}\left[\left(k_{3}+k_{4}+k_{5}\right) A_{2}-k_{5}^{2}\left(m_{2} p^{2}+\right.\right. \\
& \left.\left.+k_{2}+k_{3}\right)-k_{3}^{2}\left(m_{1} p^{2}+k_{1}+k_{2}+k_{5}\right)-2 k_{2} k_{3} k_{5}\right](19, ")
\end{aligned}
$$

In turn:

$$
W_{B}(p)=\frac{\tilde{R}_{\| l}}{\tilde{Q}_{3}}=\frac{k_{4} A_{2}}{A_{0}} .
$$

Generally from (19"') and $\left(19^{\mathrm{IV}}\right)$ follows that peak-frequency characteristics have on three resonances, and also two frequencies on which dynamic responses $\tilde{R}_{m_{3}}$ will be equal to zero. It corresponds to a mode when the dynamic part of the general response on object of protection $\mathrm{m} 3$ will be equal to zero, but there is a loading static, defined by gravity of elements vibroprotection systems and preliminary preloads springs. As to dynamic response to a basic surface $I I$ according to $\left(19^{\mathrm{IV}}\right)$ response $R_{I I}$ will have also two frequencies «zeroing». We will notice that these modes coincide with modes of dynamic clearing on co-ordinate $\mathrm{y}_{3}$ at action on object of protection of force $Q_{3} \neq 0\left(Q_{2}=0, z_{1}=0, z_{2}=0\right)$ that follows from equation (12) or (18).

Special cases are in the present state of affairs possible, for example, $\mathrm{k}_{4}$ and $\mathrm{k}_{5}$ can be accepted equal to zero. In this case equation (19) will be transformed to a kind:

$$
\tilde{k}_{c 1}^{\prime}=\frac{k_{3} A_{3}-k_{3}^{2}\left(m_{1} p^{2}+k_{1}+k_{2}\right)}{A_{3}},
$$

where

$$
\begin{gathered}
A_{3}=\left(m_{1} p^{2}+k_{1}+k_{2}\right)\left(m_{2} p^{2}+k_{3}\right)+ \\
+k_{2}\left(m_{1} p^{2}+k_{1}\right) .
\end{gathered}
$$

Knowing (20), it is possible to find dynamic response to object of protection $\mathrm{m} 3$ :

$$
\tilde{\mathrm{R}}_{\mathrm{m}_{3}}^{\prime}=\tilde{\mathrm{k}}_{\mathrm{np}_{1}}^{\prime} \tilde{\mathrm{y}}_{3}^{\prime}
$$

where $\tilde{y}_{3}^{\prime}=W_{3}(p) \tilde{Q}_{3} \quad\left(k_{4}=0, k_{5}=0\right)$.

Thus, for object of protection dynamic responses both for contacts to a basic surface, and for the full response representing the sum static and dynamic component can be found. If $k_{4}=0, k_{5}=0$, value of response also can be found at use (20).

2. To find dynamic response to intermediate weight $\mathrm{m} 1 \mathrm{VPD}$, it is necessary to use equation (16):

$$
\begin{aligned}
& W_{1}(p)=\frac{\tilde{y}_{1}}{\tilde{Q}_{3}}=\frac{\left|k_{2} k_{3}+k_{5}\left(m_{2} p^{2}+k_{2}+k_{3}\right)\right| / A_{4}}{\left(m_{1} p^{2}+k_{1}+k_{2}+k_{5}\right)-\frac{k_{5}^{2}\left(m_{2} p^{2}+\right.}{A_{4}} \ldots} \\
& \left.+k_{2}+k_{3}\right)+\left(m_{3} p^{2}+k_{3}+k_{4}+k_{5} k_{2}^{2}-2 k_{2} k_{3} k_{5}\right.
\end{aligned}
$$

where

$$
A_{4}=\left(m_{2} p^{2}+k_{2}+k_{3}\right)\left(m_{3} p^{2}+k_{3}+k_{4}+k_{5}\right)-k_{3}^{2} .
$$


The structural scheme for a case of reduction of the general system to an intermediate element $\mathrm{m} 3$ looks like, as is shown in fig. 4.

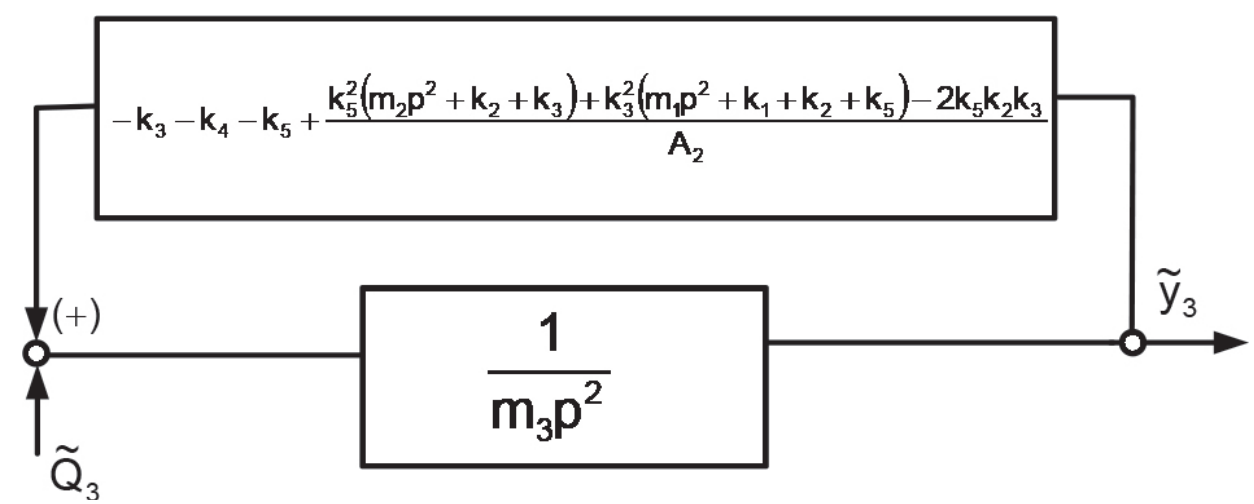

Fig. 4. Structural scheme for identification of dynamical response on intermediate element of VPD - m 1

Let's notice that at identification of transfer function (23) it is possible to present some external force $\tilde{Q}_{1}^{\prime}$ which looks like on a system input:

$$
\tilde{\mathrm{Q}}_{1}^{\prime}=\tilde{\mathrm{Q}}_{1}^{\prime} \frac{\mathrm{k}_{2} \mathrm{k}_{3}+\mathrm{k}_{5}\left(\mathrm{~m}_{2} \mathrm{p}^{2}+\mathrm{k}_{2}+\mathrm{k}_{3}\right)}{\mathrm{A}_{4}} .
$$

In this case $\tilde{Q}_{1}^{\prime}$ is the equivalent force of external indignation transferred from object of protection $\mathrm{m}_{3}$ to intermediate weight VPD $-\mathrm{m}_{1}$. In turn, if to consider that indignation in system looks like (25) it is possible to define transfer function from $\tilde{Q}_{1}^{\prime}$ on coordinate $\tilde{y}_{3}$. The coerced stiffness of the generalized spring, and that is a physical essence of a chain of negative feedback in the structural scheme on fig. 4 , is defined (at $\mathrm{k} 5=0$ ):

$$
\tilde{\mathrm{k}}_{\mathrm{c}}^{\prime \prime}=\frac{\left(\mathrm{k}_{1}+\mathrm{k}_{2}\right) \mathrm{A}_{5}-\mathrm{k}_{2}^{2}\left(\mathrm{~m}_{3} \mathrm{p}^{2}+\mathrm{k}_{3}+\mathrm{k}_{4}\right)}{\mathrm{A}_{5}}
$$

where

$$
A_{5}=\left(m_{2} p^{2}+k_{2}+k_{3}\right)\left(m_{3} p^{2}+k_{3}+k_{4}\right)-k_{3}^{2} .
$$

As to response identification $\widetilde{R}_{I}$ it can be found

$$
\tilde{R}_{\mathrm{I}}=\mathrm{k}_{1} \tilde{\mathrm{y}}_{1}=\mathrm{k}_{1} \mathrm{~W}_{1}^{*}(p) \tilde{\mathrm{Q}}_{1},
$$

here $W_{1}^{*}(p)$ it is defined from $(16)$ at $k 5=0$; that is $W_{1}^{*}(p)=W_{1}(p)_{k_{5}=0}$.

Dynamic response $\widetilde{R}_{I}$ is defined by level of interaction with a basic surface I (fig. 1). Response to an intermediate element can be found 


$$
\tilde{R}_{m_{1}}(p)=\tilde{k}_{c}^{\prime \prime} \tilde{y}_{1}=\frac{\left(k_{1}+k_{2}\right)\left[\left(\begin{array}{l}
m_{2} p^{2}+ \\
+k_{2}+k_{3}
\end{array}\right) \cdot\left(\begin{array}{l}
m_{3} p^{2}+ \\
+k_{3}+k_{4}
\end{array}\right)-k_{3}^{2}\right]-k_{2}^{2}\left(\begin{array}{c}
m_{3} p^{2}+ \\
+k_{3}+k_{4}
\end{array}\right)}{A_{5}} W_{1}^{*}(p) \tilde{Q}_{1}^{\prime} .
$$

For identification $W_{m_{1}}(p)$ it is entered with the account $\widetilde{Q}_{1}^{\prime}$, defined (25), and accepting $\mathrm{k}_{5}$ $=0$.

Similarly on dynamic response to a basic surface $I$ and an intermediate element $\mathrm{m}_{1}$ transfer functions from external force $\widetilde{Q}_{3}$ on parameters of responses $\widetilde{R}_{\mathrm{l}}$ and $\tilde{\mathrm{R}}_{\mathrm{m}}$, and also

transfer functions on corresponding dynamic responses at power entrance influence $\widetilde{Q}_{3}$ can be received.

3. Let's pass to identification of dynamic response from $Q_{3}$ on coordinate $y_{2}$, connected with intermediate weight $m_{2}$. In this case equation (17) becomes:

$$
W_{2}(p)=\frac{\tilde{y}_{2}}{\tilde{Q}_{3}}=\frac{\frac{\left[k_{5}+k_{2}+k_{3}\left(m_{1} p^{2}+k_{1}+k_{2}+k_{5}\right)\right]}{A_{6}}}{\left(m_{2} p^{2}+k_{2}+k_{3}\right)-\frac{\left(\begin{array}{l}
m_{1} p^{2}+k_{1}+ \\
+k_{2}+k_{5}
\end{array}\right) k_{3}^{2}}{A_{6}}-\frac{\left(\begin{array}{l}
m_{3} p^{2}+k_{3}+ \\
+k_{4}+k_{5}
\end{array}\right) k_{2}^{2}+2 k_{2} k_{3} k_{5}}{A_{6}}}
$$

where

$$
A_{6}=\left(m_{1} p^{2}+k_{1}+k_{2}+k_{5}\right)\left(m_{3} p^{2}+k_{2}+k_{3}\right)-k_{5}^{2}
$$

The structural scheme of system for an intermediate element $\mathrm{m} 2$ is resulted on fig. identification of dynamic response from $\mathrm{Q}_{3}$ on 5 .

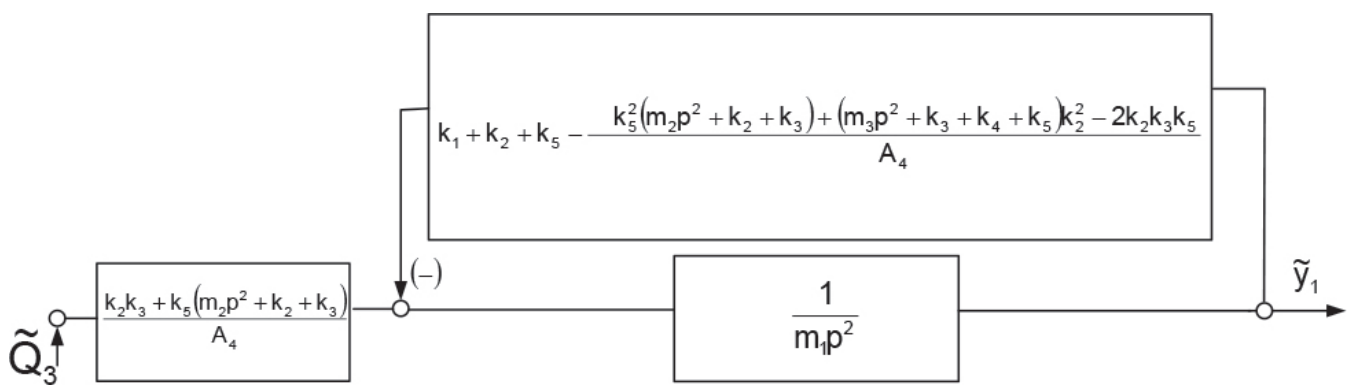

Fig. 5. Coerced structural scheme for identification of dynamicalresponse from $\tilde{Q}_{3}$ on coordinate $\tilde{\mathrm{Y}}_{2}$

The coerced stiffness of the generalized spring is defined under the scheme on fig. 5 ; at $\mathrm{k} 5=0$

$$
\tilde{\mathrm{k}}_{\mathrm{c}}^{\prime \prime}=\frac{\left(\mathrm{k}_{2}+\mathrm{k}_{3}\right) \mathrm{A}_{7}-\mathrm{k}_{3}^{2}\left(\mathrm{~m}_{1} \mathrm{p}^{2}+\mathrm{k}_{1}+\mathrm{k}_{2}\right)-\left(\mathrm{m}_{3} \mathrm{p}^{2}+\mathrm{k}_{3}+\mathrm{k}_{4}\right) \mathrm{k}_{2}^{2}}{\mathrm{~A}_{7}}
$$

where

$$
A_{7}=\left(m_{1} p^{2}+k_{1}+k_{2}\right)\left(m_{3} p^{2}+k_{3}+k_{4}\right) .
$$


Let's find values $\tilde{\mathrm{k}}_{\mathrm{c}}^{\prime}, \tilde{\mathrm{k}}_{\mathrm{c}}^{\prime \prime}, \tilde{\mathrm{k}}_{\mathrm{c}}^{\prime \prime \prime}$ directly from the design scheme on fig. 1 at $\mathrm{k} 5=0$, using a technique stated in [4], and we will receive:

$$
\begin{aligned}
& \tilde{k}_{c}^{\prime *}=k_{4}+\frac{\left.k_{3} \mid\left(m_{1} p^{2}+k_{1}\right) k_{2}+m_{2} p^{2}\left(m_{1} p^{2}+k_{1}+k_{2}\right)\right]}{\left(k_{1}+m_{1} p^{2}\right) k_{2}+m_{2} p^{2}\left(m_{1} p^{2}+k_{1}+k_{2}\right)+k_{3}^{2}\left(m_{1} p^{2}+k_{1}+k_{2}\right)} \\
& \tilde{k}_{c}^{\prime \prime *}=k_{1}+\frac{k_{2}\left[\left(m_{3} p^{2}+k_{4}\right) k_{3}+m_{2} p^{2}\left(m_{3} p^{2}+k_{3}+k_{4}\right)\right]}{\left(k_{4}+m_{3} p^{2}\right) k_{3}+m_{2} p^{2}\left(m_{3} p^{2}+k_{3}+k_{4}\right)+k_{2}\left(m_{3} p^{2}+k_{3}+k_{4}\right)} \\
& \tilde{k}_{c}^{\prime \prime * *}=\frac{\left.k_{2}\left[\left(m_{1} p^{2}+k_{1}\right)\left(m_{3} p^{2}+k_{3}+k_{4}\right)\right)+k_{3}\left(m_{1} p^{2}+k_{1}+k_{2}\right)\left(m_{3} p^{2}+k_{4}\right)\right]}{\left(k_{1}+k_{2}+m_{1} p^{2}\right)\left(m_{3} p^{2}+k_{3}+k_{4}\right)}
\end{aligned}
$$

III. The method of straight transformations of design scheme. Doing corresponding transformations to equations (19) at $\mathrm{k} 5=0$ (31), (35), it is possible to show that the equations received on the basis of the offered method and at use of ways of direct convolution, coincide. Design schemes for reception coerced stiffness's are resulted on fig. 6a,b.

In particular, on fig. 6 and the elastic element with stiffness $\mathrm{k} 4$ enters into a chain consistently connected elastic and mass-

a)

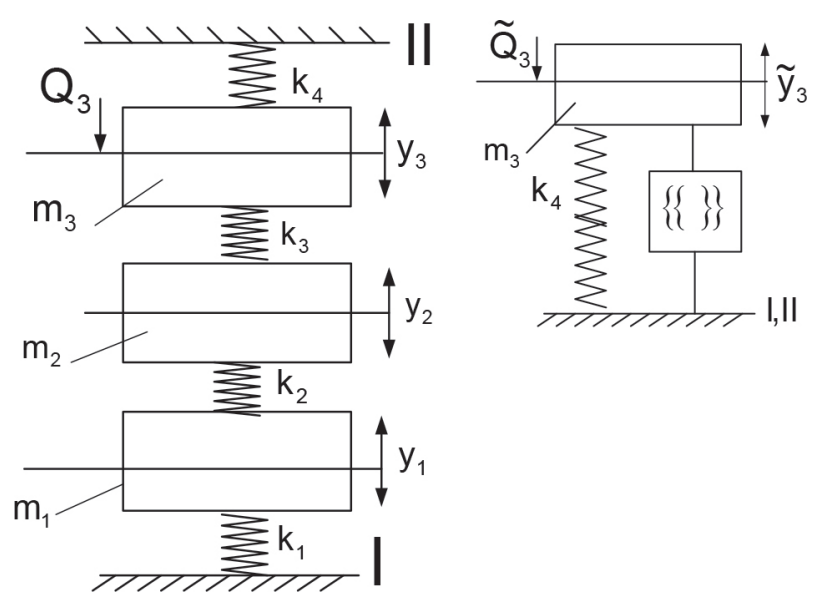

Fig. 6. Principal scheme of formation of coerced stiffness on design scheme (a) at force disturbance $\widetilde{\mathbf{Q}}_{3}$ on protection object; b) coerced scheme

The coerced stiffness $\{\{\}\}$ in this case is formed in the following sequence of actions: inertial elements between basic surfaces I and II (k1, m1, k2, m2, k3, m3, k4). By transformations the design scheme on fig. 6 and can be transformed to system with one degree of freedom containing (fig. 6 b) the object of protection leaning against the general basic surface I, II. Thus the system is led to a kind of parallel connection from two elastic elements. One of them is a spring with stiffness $\mathrm{k} 4$, and the second represents a mechanical chain with the coerced stiffness $\{\{\}\}$. b) 


$$
\left(m_{1} p^{2}\right) \rightarrow\left[\frac{\left(m_{1} p^{2}+k_{1} k_{2}\right.}{m_{1} p^{2}+k_{1}+k_{2}}\right] \rightarrow\left\{[]+m_{2} p^{2}\right\} \rightarrow\left\{\left\{\frac{\left\{k_{3}\right.}{\{\}+k_{3}}\right\}\right\}
$$

which leads to equation (34).

On fig. 7 a, b it is shown, how the coerced stiffness is formed, that is the factor of stiffness of the generalized spring (34) is defined. It is

connected with identification of equivalent external force $\tilde{Q}_{1}^{\prime}$ under the formula (25) that is resulted on fig. 4 .

a)

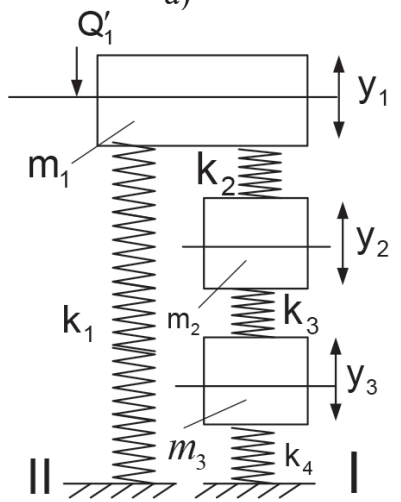

b)

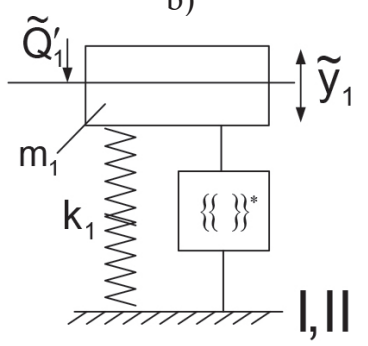

Fig. 7. Principal scheme of formation of coerced stiffness on design scheme (a) at equivalent force disturbance $\widetilde{\mathbf{Q}}_{1}^{\prime}$ on element $m_{1}$; b) coerced scheme

At identification of the coerced stiffness of the generalized spring $\{\{\}\} * *$ (fig. $7 \mathrm{~b}$ ) is used the following sequence of operation of convolution of structure

$$
\left(m_{3} p^{2}+k_{4}\right) \rightarrow\left[\frac{\left(m_{3} p^{2}+k_{4} k_{3}\right.}{m_{3} p^{2}+k_{3}+k_{4}}\right] \rightarrow\left\{[]+m_{2} p^{2}\right\} \rightarrow\left\{\left\{\frac{\left\{k_{2}\right.}{\{\}^{*}+k_{2}}\right\}\right\}^{*}
$$

which leads to equation (35) that is schematically represented on fig. 7 b. The general stiffness is defined by summation $\mathrm{k} 1$ and $\{\{\}\} *$.
As to identification of the coerced stiffness connected with an intermediate element $\mathrm{m} 2$, equivalent force (fig. 5) it is defined by equation

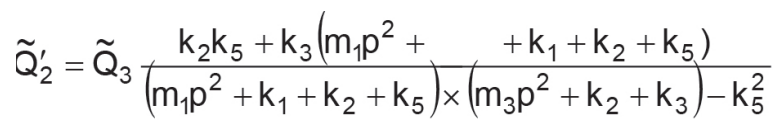

At the further calculations it is accepted $\mathrm{k} 5=0$. $8 \mathrm{a}, \mathrm{b}$.

The basic design scheme and reduction possibility to the simplified kind are reflected on fig. 
a)

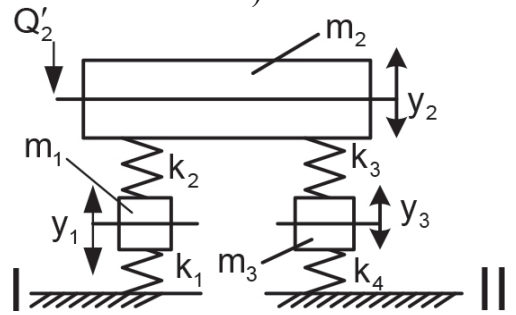

b)

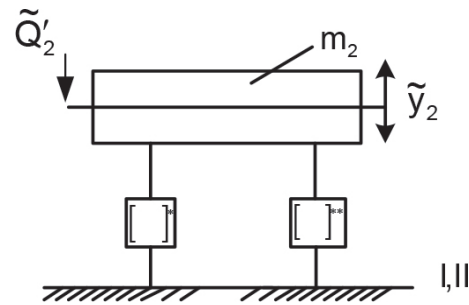

Fig. 8. Principal scheme of formation of coerced stiffness of generalized spring on design scheme $(a) ; b)$ coerced scheme

In this case equivalent external force $\tilde{Q}_{2}^{\prime}$ is defined from equation (36). In turn the general factor of stiffness is formed by the sum of two stiffness's []* and []**(fig. 8 b), on the basis of realization of following sequences of actions:

$$
\left(m_{1} p^{2}+k_{1}\right) \rightarrow\left[\frac{\left(m_{1} p^{2}+k_{1}\right) k_{2}}{m_{1} p^{2}+k_{1}+k_{2}}\right]^{*} \text { and also }\left(m_{3} p^{2}+k_{4}\right) \rightarrow\left[\frac{\left(m_{3} p^{2}+k_{4}\right) k_{3}}{m_{3} p^{2}+k_{3}+k_{4}}\right]^{* *}
$$

that finally, leads to equation (38).

Thus, definition of dynamic responses is connected with an estimation resulted stiffness's the generalized springs which create dynamic the rigidity, depending on frequency of external revolting influences. Two approaches based on the offered method of formation of a chain of return negative communication on allocated mass-inertial an element, and also on procedures of consecutive convolution with use of initial settlement schemes (a Fig. 6a, 7a, 8 a) are shown. We will notice that convolution is simple enough only for planar systems, therefore in this case was accepted that.

When non-planarity it is necessary to take into consideration, it is necessary to use a number of preliminary transformations that complicates a technique of definition resulted жесткостей, but leads to similar results.

Conclusions. Thus a basis of a method of definition of dynamic reactions on object of protection, and also on inertial elements vibroprotection devices (B3Y) and on basic surfaces I and II is transformation of the block diagramme of system to a kind corresponding to a link of integration of the second order with formation of additional negative feedback which, inherently, and represents dynamic reaction. In system with three degrees of freedom such transformations become in case of need not only for object of protection, but also for intermediate weights. The method is applicable and for systems with a great number of degrees of freedom, however, thus there is an appreciable growth of labour input of calculations. The structure of additional negative feedback can be received more simple way if to use the characteristic equation of system entering into corresponding transfer function. The equation is transformed with allocation necessary partial systems with the subsequent transformations of structure.

At construction of the resulted block diagrams carrying over of the force enclosed initially to object of protection, to an intermediate inertial element can be carried out. Such force is equivalent in the sense that the transfer functions received with use, at carrying over of force to a new point yield that result, as at a usual order. The offered method allows to receive transfer function of dynamic response of an element or a support by multiplication of the corresponding resulted factor of rigidity of the generalized spring (or dynamic rigidity) on corresponding displacement of an element in system of co-ordinates. 


\section{REFERENCES}

1. Artobolevskiy, I.I. Theory of mechanisms and machines. - M.: Science. 1975. - 638 $p$.

2. Belokobil'skiy, S.V., Eliseev, S.V., Kashuba, V.B. Applied tasks of the structural theory of vibroprotective systems. - SPb.; Politekhnika, 2013. - $364 \mathrm{p}$.

3. Eliseev, S.V., Artyunin, A.I., Ermoshenko, Yu.V. and etc. Method of transformation of mechanical oscillation systems on base of introduction in connection of intermediate devices / ISTU. - Irkutsk, 2014. - 68 p. - Ref . : 25 names. - Rus. - Dep . in VINITI 14.01.14 № $19-$ V 2014

4. Eliseev, S.V., Kargapol'cev, S.K. Display of features of connections of mechanical elements in mechanical chains theory / Materials of All-Russian science conference "Information technologies in science and control». Irkutsk. 2014. pp. 100-108

5. Eliseev, S.V., Moskovskikh, A.O., Kaimov, E.V. Structural interpretations of mechanical oscillation systems in electrical chains theory and automation control theory; ISTU. - Irkutsk, 2014. - 82 p. Dep. in VINITI 13.01.2014 №8 - V 2014.

6. Eliseev, S.V., Reznik, Yu. N., Khomenko, A.P., Zasyadko, A.A. Dynamic synthesis in the generalized problems of vibroprotection and a vibration insulation of technical objects. - Irkutsk: publ. ISU, 2008. - 523 p.

7. Frolov, K.V., Popov, S.A., Musatov, A.K. and etc. Theory of mechanisms and machines: textbook for universities. - M.: High school. 1978. - $496 p$.

8. Khokhlov, A.A. Dynamics of complicated of mechanical systems. - M.: MIIT. 2002. $172 \mathrm{p}$.

9. Kolchin, N.I.. Machines mechanics: in 2 v. Ed. thied rec. L.:Mechanical engineering, 1971. V.2. $-456 p$.

10. Korolkov, M.V. Development and research of analytical models of dynamics of mechanisms with gaps in couplings of details: 05.02.02 / Korolkov Mikhail Vladimirovich. MADI.- Moscow, 2010.- 176 p.

11. Levitskiy, N.I. Oscillations in mechanisms / N.I. Levitskiy. - M.: Science, 1988. 358 $p$.

12. Lurie, A.I. Analytical mechanicsa. - M.: Science, 1968. -720 p.

13. Vul'fson, I.I. Dynamics of mechanisms with accounting of links elasticity. L.: Ed. LPI of M.I. Kalinina, 1984. - 40 p. 